\title{
Effective waveform representation of responsive signals initiated by impulsive actions in physical and engineering systems \\ DOI:
}

10.1016/j.jengsci.2019.103199

\section{Document Version}

Accepted author manuscript

Link to publication record in Manchester Research Explorer

Citation for published version (APA):

Yan, Y., \& Li, Q. M. (2020). Effective waveform representation of responsive signals initiated by impulsive actions in physical and engineering systems. International Journal of Engineering Science, 147, [103199].

https://doi.org/10.1016/j.ijengsci.2019.103199

\section{Published in:}

International Journal of Engineering Science

\section{Citing this paper}

Please note that where the full-text provided on Manchester Research Explorer is the Author Accepted Manuscript or Proof version this may differ from the final Published version. If citing, it is advised that you check and use the publisher's definitive version.

\section{General rights}

Copyright and moral rights for the publications made accessible in the Research Explorer are retained by the authors and/or other copyright owners and it is a condition of accessing publications that users recognise and abide by the legal requirements associated with these rights.

\section{Takedown policy}

If you believe that this document breaches copyright please refer to the University of Manchester's Takedown Procedures [http://man.ac.uk/04Y6Bo] or contact uml.scholarlycommunications@manchester.ac.uk providing relevant details, so we can investigate your claim.

\section{OPEN ACCESS}




\title{
Effective waveform representation of responsive signals initiated by impulsive actions in physical and engineering systems
}

\begin{abstract}
We report applications of a signal decomposition method based on a basic shock waveform to a wide range of dynamical response signals initiated by impulsive actions in physical and engineering systems. The generality and significance of the shock waveform are demonstrated for time-series signals in different physical and engineering systems disturbed by impulsive actions due to various causes. Case examples include, but are not limited to, signals of heartbeat, earthquake, gravitational wave of binary coalescence, Enhanced Long Range Navigation System (Loran), and mechanical and pyrotechnic shocks. The simplicity of the shock waveform can facilitate the understanding of the intrinsic pattern of the signal and the intrinsic characteristics of the system.
\end{abstract}

Keywords: Dynamical System, Heart Sound, Earthquake, Gravitational Wave

\section{Introduction}

Data recorded in time series format contains rich information, whose pattern, however, can only be understood with proper means such as Fourier transform, Prony analysis or wavelet transform using wellselected bases[1-3].

The particular expressions of a general waveform $a t^{n-1} e^{-b t} \cos (\omega t+\phi)$ termed Gammatone due to its link to the Gamma distribution[4], have been realized in several engineering disciplines. In the Gammatone expression, $a$ is the amplitude, $b$ is the bandwidth, the order $n$ is a positive integer, $\omega$ is the center frequency and $\phi$ is the phase. It has been used extensively in auditory discipline for sound signal processing and sound filtering through cochlear $(n=3$ and 4$)[5,6]$. Up to now, it was only used sporadically in other engineering disciplines, i.e. $n=2$ for mechanical shock and earthquake synthesis $[7,8], n=3$ for hyperbolic radio navigation (Loran-C signal)[9]. To analyse more complex mechanical shocks, 'shock waveform' (SW) as a basis was proposed based on a linear elastic multi-degree of freedom model[10], which is mathematically the same as the Gammatone[4], but has different expressions. The SW well matches the characteristics of the system response initiated by an impulsive action and can also work as a dictionary in matching pursuit algorithm to decompose the system's response sparsely[11]. The parameters in SW expression have clear physical meanings and can be linked to physical mechanisms. For instance, a mechanical shock can be divided into three categories, i.e., near-, mid- and far-field shock, according to the peak time parameter[10].

\section{Shock Waveform Representation}

The SW function proposed in [10] can be extended symmetrically about $\tau=0$ to the whole real field

$$
w(t)= \begin{cases}A t^{\zeta \omega \tau} \tau^{-\zeta \omega \tau} e^{\zeta \omega(\tau-t)+i(\omega t+\varphi)} H(t), & \tau \geq 0 \\ A t^{-\zeta \omega \tau} \tau^{\zeta \omega \tau} e^{\zeta \omega(t-\tau)+i(\omega t+\varphi)}(1-H(t)), & \tau<0\end{cases}
$$

where $A$ is the peak amplitude of the waveform, $\omega$ and $\phi$ have been defined, $\zeta$ is the damping ratio, $\tau$ is the peak time at which the amplitude of SW is $A, i$ is the imaginary unit, and $H(t)$ is the Heaviside step function. 
a
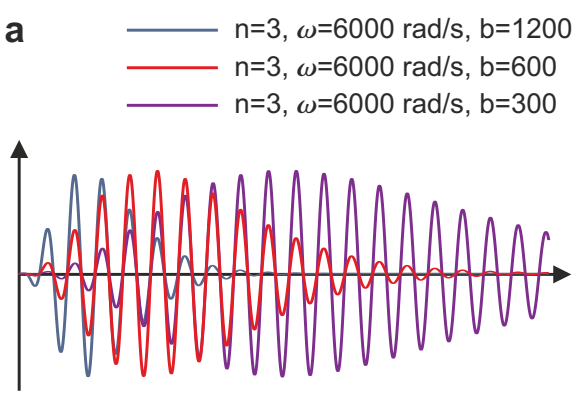

b

$\tau=5 \mathrm{~ms}, \omega=6000 \mathrm{rad} / \mathrm{s}, \zeta=0.4$

$\tau=5 \mathrm{~ms}, \omega=6000 \mathrm{rad} / \mathrm{s}, \zeta=0.2$

$\tau=5 \mathrm{~ms}, \omega=6000 \mathrm{rad} / \mathrm{s}, \zeta=0.1$

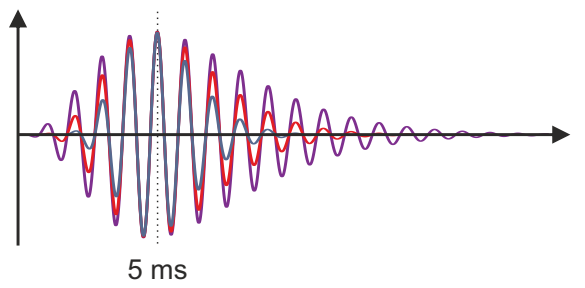

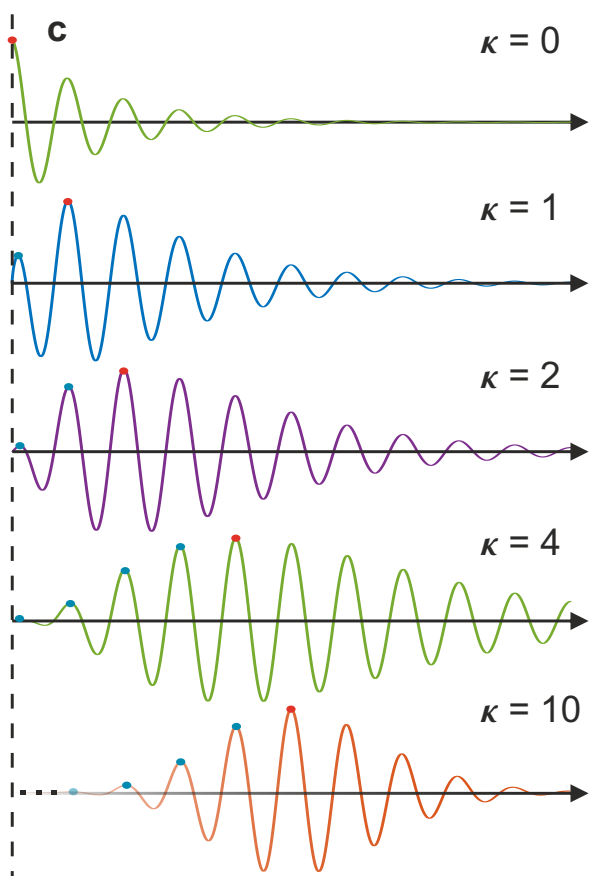

Figure 1: (a) Influence of parameter $n$ on SW; (b) Influence of parameter $\tau$ on the peak time of SW; (c) Influence of parameter $\kappa$ on the symmetry of SW.

Parameters in SW have clearer physical meanings than those in Gammatone expression. For example, the order parameter $n$ in Gammatone determines the overall shape of the waveform jointly with other frequency and damping parameters (Fig.1A) while the parameter $\tau$ in Eq.1 represents the peak time of the waveform (Fig.1B) regardless of its frequency and damping parameters. Considering the period of the carrier harmonic wave, $\tau$ can be normalized, i.e. $\kappa=\frac{\tau \omega}{2 \pi}$, to describe the necessary periods for a SW to reach its peak amplitude, as demonstrated in Fig. $1 \mathrm{C}$ where the peak point is marked with a red dot while the local extrema points before peak point are marked with blue dots. The number of carrier wave period at the peak time is indicated by their $\kappa$ values. The parameter $|\kappa|$ can also independently describe the degree of symmetry of a SW component. If $|\kappa|$ is close to zero, SW is similar to a damped sine wave. With the increase of $|\kappa|$, the SW is changed from damped sine wave to common wavelet (i.e. in the range of transition), and has an asymmetric envelope. When $|\kappa|$ value is sufficiently large, e.g., higher than 10 , the envelope of SW becomes more symmetric and the SW approaches to common wavelet. For a mechanical shock, values of parameter $|\kappa|$ can be used to quantitatively classify the near-, mid- and far-field shocks[10].

For a given time series signal, $r(t)$, the reconstructed signal $\hat{r}(t)$ is determined by

$$
\hat{r}(t)=\sum_{i=1}^{m} T_{i}\left(w_{i}(t)\right)
$$

where $m$ is an integer determined later; $T_{i}\left(w_{i}(t)\right)$ is a temporally translated (or shifted) SW component, i.e.,

$$
T_{i}\left(w_{i}(t, \tau, A, \omega, \zeta, \varphi)\right)=w_{i}\left(t-\stackrel{\circ}{t}_{i} \tau-\stackrel{\circ}{t}_{i}, A, \omega, \zeta, \varphi\right)
$$

where $\dot{t}_{i}$ is the shifted initial time (from zero) of the $i$ th SW component. $m$ SW components are used to reconstruct signal, in which the coefficient of determination $\left(R^{2}\right)$ of the reconstructed signal is required to be sufficiently large ( $90 \%$ is used here) to ensure a good representation with the given signal. SW components are extracted and sorted in descending order of their signal energies. The decomposition procedure is stable 


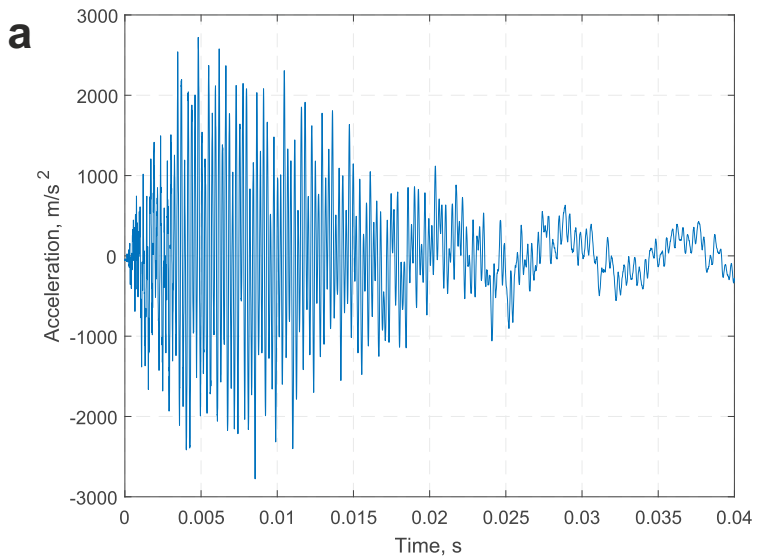

b

\begin{tabular}{|l|l|l|}
\hline Basis & $\begin{array}{l}\text { Number of } \\
\text { Terms }\end{array}$ & $\begin{array}{l}\text { Total } \\
\text { Coefficients }\end{array}$ \\
\hline $\begin{array}{l}\text { Shock } \\
\text { Waveform }\end{array}$ & 3 & 18 \\
\hline Wavelet & 12 & 48 \\
\hline $\begin{array}{l}\text { Discrete } \\
\text { Cosine II }\end{array}$ & 18 & 36 \\
\hline $\begin{array}{l}\text { Damped } \\
\text { Sinusoids }\end{array}$ & 234 & 936 \\
\hline
\end{tabular}

Figure 2: Decomposition efficiency with various basis. (a) Example mechanical shock signal[10]. (b) Number of terms and the total coefficients needed for signal reconstruction.

under small changes of the input signal, which normally has small signal energy and is discarded as noise or unimportant information. The treatment of SW and the signal decomposition procedure can be found in Appendix A.

Since SW is an over-complete dictionary, it can be used to analyse all kind of signals. However, SW is especially good at describing asymmetric non-stationary signal between a damped sine wave and a common (symmetric) wavelet, in which a signal can normally be represented by SW sparsely if the $\kappa$ values of signal's dominant components are between 0 and 10. Using a mechanical shock signal as an example, Fig.2a show the acceleration-time history with 4000 samples, which is decomposed by various common basis functions. Fig. $2 \mathrm{~b}$ shows the necessary number of terms and coefficients to reconstruct signals and meet the $90 \% R^{2}$ requirement. In comparison to SW basis, discrete cosine transform-II basis[12], damped sinusoids basis[13] and NASA's wavelet basis[14] are also used. SW is the most efficient basis for the decomposition of this kind of signals, that only needs 18 coefficients of 3 terms to describe the original signal. The wavelet and discrete cosine II bases have similar performance. Since the coefficient numbers for each term are different, wavelet decomposition needs less terms but more total coefficients than the discrete cosine II does. The damped sinusoid basis with Prony's method is the least efficient way in this case, which need 936 coefficients of 234 terms for signal reconstruction.

\section{Interdisciplinary Application}

Here, we use three examples in heart sound, seismic wave and gravitational wave to demonstrate the applications of the SW decomposition to different physical and engineering systems.

\subsection{Heart Sound Signal Analyses}

The heart sound signal can be well decomposed by SW. We analysed heart sound signals from PASCAL dataset[15], which was gathered from the general public. The fundamental heart sound normally includes the first (S1) and the second (S2) heart sounds, which reflect the vibration of the entire cardiac system caused by the opening and closure of the heart valves[16].

The analytical results are listed in Fig. 2 and Table 1. Only the results for one cardiac cycle from both normal and abnormal heart sounds are demonstrated for the sake of brevity, whose time series data are plotted in Fig.3A and Fig.3D, respectively. The waveforms of both S1 (Fig.3B) and S2 (Fig.3C) in normal heart sound can be described by SW very well, i.e. only one SW component can accounts for over $90 \%$ of the variability of the sample points ( $R 2$ requirement). Although the shapes of the SW of S1 and S2 are visually different, they are both in the transition range between damped sine waves and common wavelets, which are reflected by their similar $\kappa$ values of 1.36 and 1.09, respectively. However, the abnormal murmur 


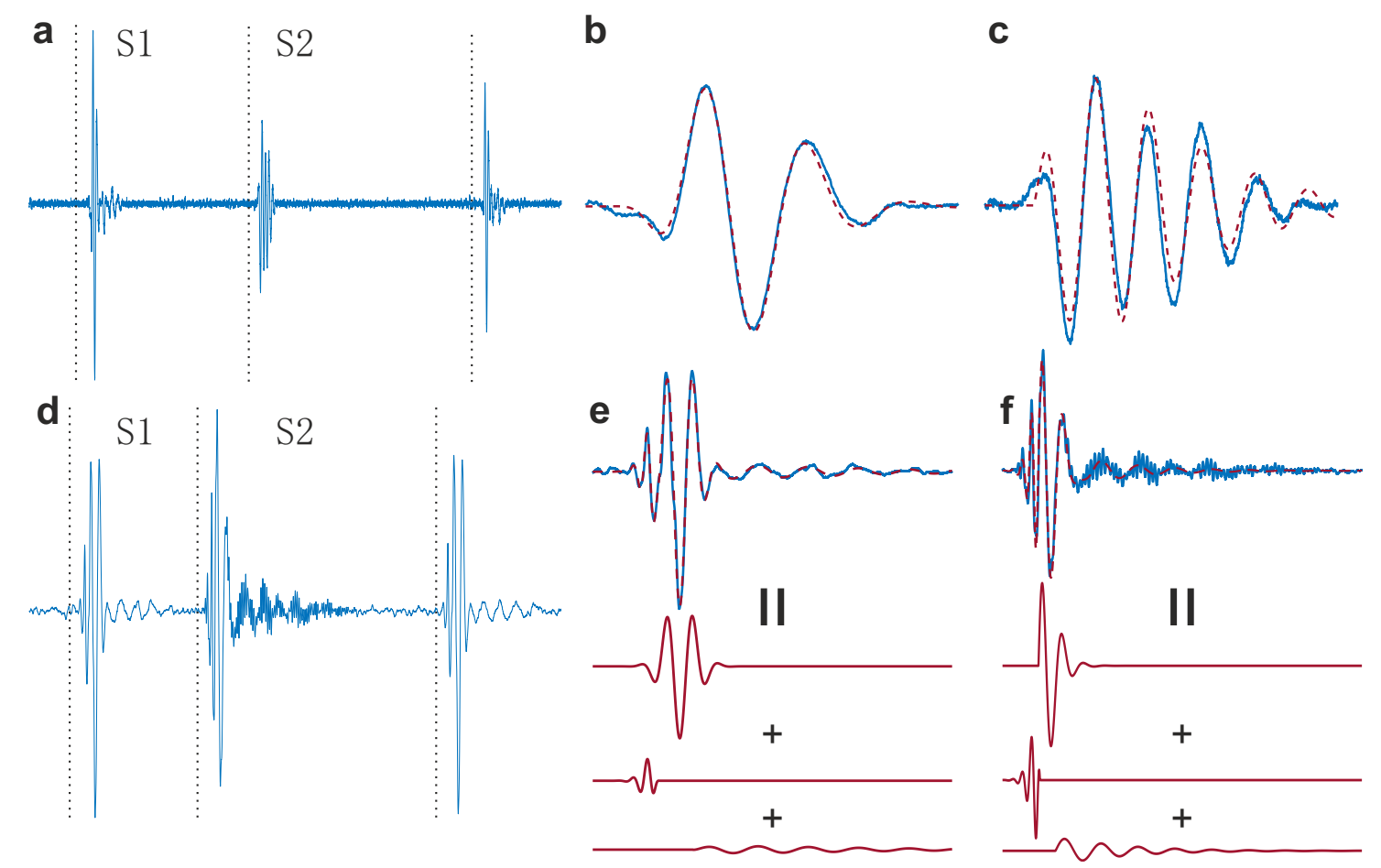

Figure 3: Heart sound analysis with the SW; lines in French blue are measured signals; lines in Maroon are SW components. (a) Normal heart sound. (b) S1 of normal heart sound. (c) S2 of normal heart sound. (d) Abnormal (murmur) heart sound. (e) S2 of abnormal heart sound. (f) SW components for S2 of abnormal heart sound.

Table 1: Table of parameters used in reconstructing normal and abnormal heart sounds.

\begin{tabular}{llllllll}
\hline Amplitude & Frequency $(\mathrm{Hz})$ & $\dot{t}_{i}(\mathrm{~ms})$ & $\tau_{i}(\mathrm{~ms})$ & $\zeta_{i}$ & $\phi_{i}$ & $\kappa_{i}$ & Comments \\
\hline-0.093 & 139.221 & 4.968 & 9.790 & 0.902 & 2.552 & 1.363 & S1 of normal heart sound \\
\hline 0.039 & 155.084 & 5.883 & 7.070 & 0.138 & 5.292 & 1.097 & S2 of normal heart sound \\
\hline-0.581 & 41.319 & -325.669 & 404.629 & 8.427 & 1.786 & 16.719 & \\
-0.179 & 75.013 & 61.050 & -10.323 & 0.770 & 2.338 & -0.774 & S1 of abnormal heart sound \\
0.028 & 21.888 & 90.966 & 42.636 & 0.084 & 4.446 & 0.933 & \\
\hline 0.653 & 34.185 & 48.300 & 9.046 & 0.484 & 5.605 & 0.309 & \\
0.373 & 80.051 & 50.353 & -6.130 & 0.414 & 5.845 & -0.491 & S2 of abnormal heart sound \\
-0.078 & 20.055 & 70.500 & 8.861 & 0.110 & 1.550 & 0.178 & \\
\hline
\end{tabular}

heart sound signals, i.e. S1 and S2 in Fig.3E and Fig.3F, respectively, have different and more complex characters after being decomposed into SW components. They both consist of three SW components, which are a main oscillation, a secondary oscillation with reduced amplitude but higher frequency, and a decaying oscillation with lower frequency. With the extraction of these patents, correlations with the dynamics of cardiac system, and therefore, the corresponding cardiac diseases, could be further studied, and automated segmentation and classification methods can be developed for clinical diagnosis.

\subsection{Seismic Wave Analyses}

Seismic wave can also be considered as the response of the earth media excited by a sudden and transient impulsive loading, e.g., the sudden release of stored elastic energy in the Earth's lithosphere due to the fast fracture of existing faults. We analysed the seismic waveforms from two severe events, which are the 2011 M-w 9.0 Tohoku-Oki earthquake (Fig.4A and Table 2) and the $2012 \mathrm{M}-\mathrm{w}$ 8.6 Sumatra earthquake (Fig.4C and Table 3). The broadband vertical seismic data at YSS and BTDF stations were obtained from the global seismic network IRIS[17]. 


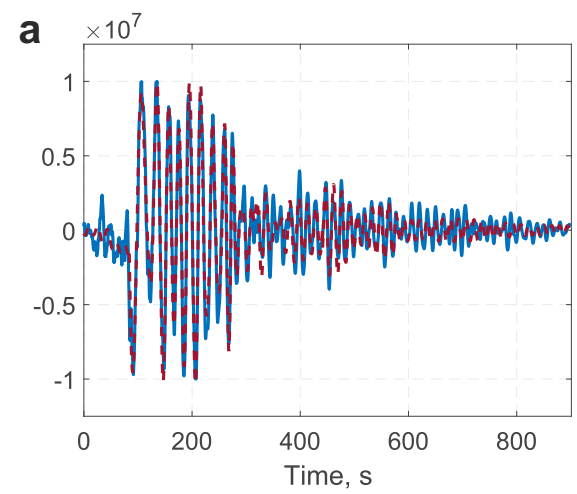

b
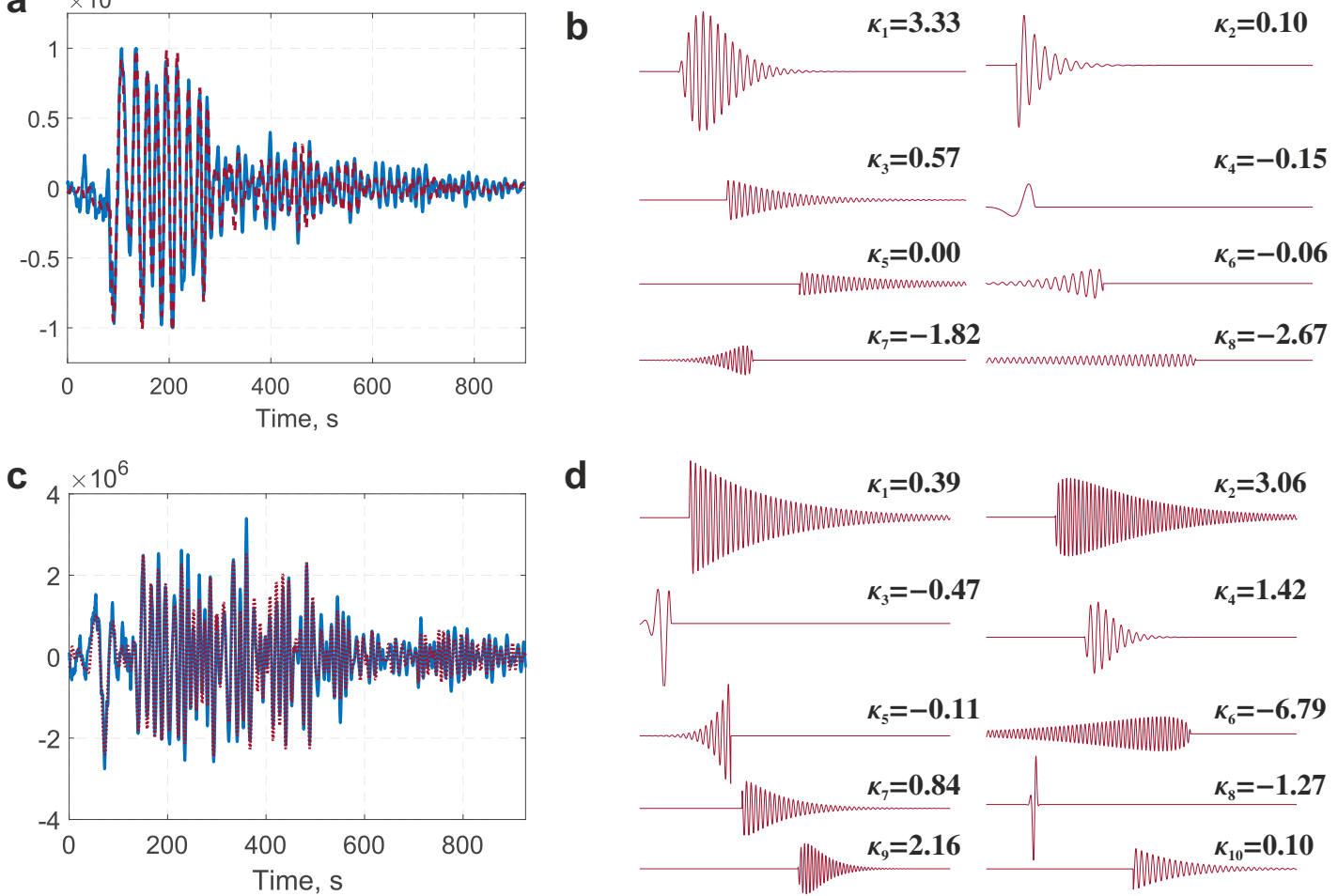

d

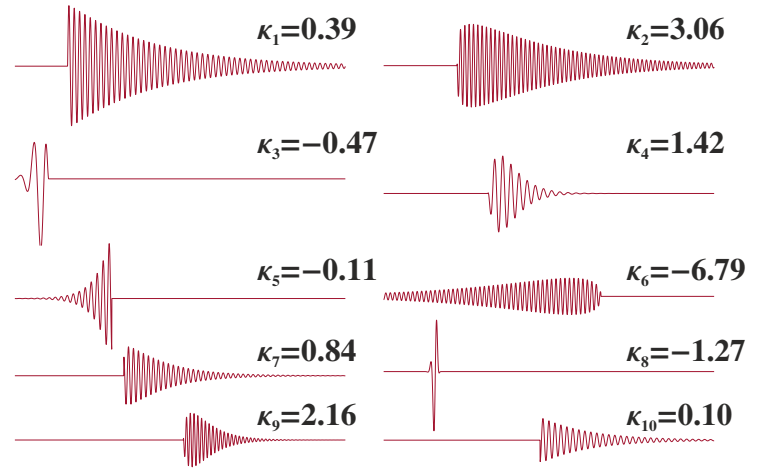

Figure 4: Seismic waves analyzed with the SW; lines in French blue are measured signals; lines in Maroon are SW reconstructed signals. (a) Vertical component seismogram of the $2011 \mathrm{M}-\mathrm{w} 9.0$ Tohoku-Oki earthquake for YSS station. (b) SW components decomposed from the Tohoku-Oki earthquake. (c) Vertical component seismogram of the 2012 M-w 8.6 Sumatra earthquake for BTDF station. (d) SW components decomposed from the Sumatra earthquake.

An earthquake signal is more complicated than a heart sound signal, which is reflected by the necessary number of SW components to meet the $R 2$ requirement. The Tohoku-Oki earthquake is constituted by eight SW components, which are listed in Fig.4B in signal energy order. The waveform shape of all SW components can be described quantitatively by their respective $\kappa$ parameters. It is obvious that this earthquake signal is mainly dominated by SW components in the forms of damped sine wave $(\kappa=0.09)$ and in the transition range $(\kappa=3.33)$. Adding all eight SW components together, the reconstructed signal meets the $90 \% R 2$ requirement as shown by the dash line in Fig.4A, which has very good agreement with the measurement signal.

Only ten among thirteen SW components extracted from the Sumatra earthquake are shown in Fig.4D, sorted according to their signal energy levels. Similar characteristics are observed in this signal, i.e. the first two SW components with the largest contributions to the overall signal are a damped sine wave and a SW in the transition range, which are reflected by their $\kappa$ values, respectively. The difference between the two earthquakes is mainly shown by their damping ratio values, which can be reflected by the duration of their dominant SW components. The excellent agreement between the reconstructed and the measured signals are shown in Fig.4C.

\subsection{Gravitational Wave Analyses}

The SW may also be applied to analyse the gravitational wave signals. The gravitational wave signals can be divided into three stages[18-20], i.e., the inspiral stage, the merger stage and the ringdown stage, where the merger happens in a rapidly-accelerated and transient way to produce much stronger gravitational wave signals, which are gradually relaxed to an equilibrium solution of the field equations in the ringdown stage. 
Table 2: Table of parameters used in reconstructing the $2011 \mathrm{M}-\mathrm{w} 9.0$ Tohoku-Oki earthquake signal.

\begin{tabular}{llllllll}
\hline Component & Amplitude $\left(\times 10^{6}\right)$ & Frequency $(\mathrm{Hz})$ & $\dot{t}_{i}(\mathrm{~s})$ & $\tau_{i}(\mathrm{~s})$ & $\zeta_{i}$ & $\phi_{i}$ & $\kappa_{i}$ \\
\hline 1 & 9.146 & 0.049 & 105.941 & 173.482 & 0.078 & 3.429 & 3.333 \\
2 & 9.636 & 0.035 & 83.948 & 86.83 & 0.083 & 1.519 & 0.101 \\
3 & 2.980 & 0.065 & 240.591 & 246.366 & 0.018 & 4.707 & 0.571 \\
4 & 5.383 & 0.006 & 136.899 & 109.807 & 2.026 & 6.203 & -0.152 \\
5 & 1.828 & 0.069 & 441.414 & 441.415 & 0.009 & 3.617 & 0.000 \\
6 & 2.626 & 0.041 & 313.063 & 311.370 & 0.038 & 6.109 & -0.068 \\
7 & 2.248 & 0.085 & 313.274 & 291.878 & 0.040 & 2.167 & -1.821 \\
8 & 0.922 & 0.060 & 573.904 & 529.094 & 0.006 & 3.971 & -2.672 \\
\hline
\end{tabular}

Table 3: Table of parameters used in reconstructing the 2012 M-w 8.6 Sumatra earthquake signal.

\begin{tabular}{llllllll}
\hline Component & Amplitude $\left(\times 10^{6}\right)$ & Frequency $(\mathrm{Hz})$ & $\dot{t}_{i}(\mathrm{~s})$ & $\tau_{i}(\mathrm{~s})$ & $\zeta_{i}$ & $\phi_{i}$ & $\kappa_{i}$ \\
\hline 1 & 1.764 & 0.067 & 147.659 & 153.606 & 0.011 & 4.627 & 0.397 \\
2 & 1.247 & 0.082 & 206.306 & 243.431 & 0.010 & 1.466 & 3.061 \\
3 & 2.200 & 0.026 & 94.565 & 76.305 & 0.542 & 0.516 & -0.471 \\
4 & 1.155 & 0.044 & 292.536 & 325.098 & 0.105 & 1.145 & 1.422 \\
5 & 1.724 & 0.060 & 271.903 & 270.038 & 0.069 & 2.788 & -0.111 \\
6 & 0.530 & 0.080 & 609.608 & 524.266 & 0.009 & 0.256 & -6.793 \\
7 & 0.843 & 0.072 & 305.514 & 317.242 & 0.023 & 0.360 & 0.839 \\
8 & 2.162 & 0.053 & 168.396 & 144.580 & 2.407 & 6.252 & -1.265 \\
9 & 0.784 & 0.095 & 472.650 & 495.516 & 0.036 & 1.054 & 2.161 \\
10 & 0.648 & 0.060 & 438.801 & 440.469 & 0.020 & 3.276 & 0.099 \\
11 & 0.418 & 0.085 & 756.653 & 756.654 & 0.002 & 4.791 & 0.000 \\
12 & 0.383 & 0.087 & 98.809 & 239.206 & 0.044 & 4.209 & 12.199 \\
13 & 0.937 & 0.034 & 188.687 & 189.599 & 0.161 & 0.354 & 0.031 \\
\hline
\end{tabular}

Similar to the natural frequency concept in mechanical vibration, gravitational wave in the ringdown stage is dominated by the quasinormal modes (QNMs) of the final product, which has the same form of damped sine wave, describing the exponential decrease of the asymmetry in time order as the product evolves towards the perfect spherical shape[21-23].

Since the energy contained in the QNMs in measurements are weak[24], we use SW to analyse the analytical ringdown and echo data from an online open dataset contributed by the Gravitation in Técnico[25, 26], which is the quasicircular inspiral of a mass ratio 1:100 binary. The gravitational signal is plotted in Fig.5A, along with the reconstructed signal with SW components. The ringdown and echo signals can be synthesized by SW components, i.e., each signal in this stage can be descripted very well with only one SW component. There are one ringdown signal in the shape of damped sine wave and five echo signals in the transition range in Fig.5A. Six SW components are needed in this case, whose parameters are shown in Fig.5B. Besides common features, i.e., decreasing of amplitude and frequency, these parameters show some interesting patterns. The $\dot{t}_{i}$ parameters give clear initial times of all waveforms, which are nearly evenlyspaced in the signal. The decay rates are described by the damping ratio $\zeta_{i}$, decreasing gradually among five echoes. The waveform shape changes from damped sine wave towards wavelet, which is characterized by the smooth growing parameter $\kappa_{i}$ from 0.15 to 3.77 . With the above parameter patterns, the SW decomposition may be considered as a heuristic method to extract ringdown signal from gravitational wave measurements, which allows for more precise measurements of the black hole mass and spin, and provides a new way to understand the gravitational wave signal.

\section{Conclusion}

It seems that the SW is an explicit and general waveform applicable to various physical and engineering systems. Six parameters in each waveform component have their corresponding physical meanings for each individual science or engineering system. Along with adaptive SW bases, a non-stationary signal with asymmetrical envelope can be represented by the sum of several SW components, showing the features 


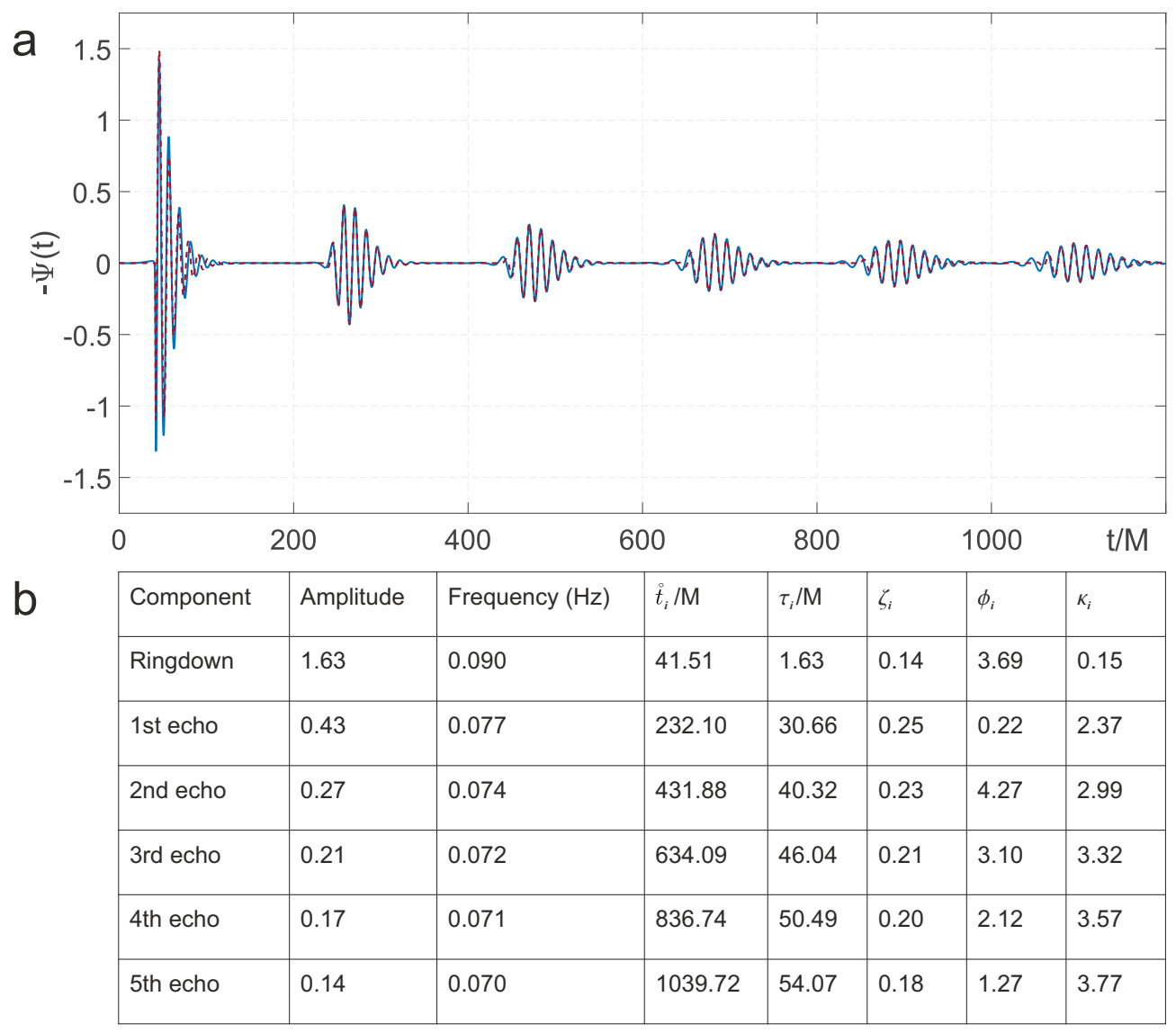

Figure 5: Ringdown and echoes of gravitational wave analysed with the SW decomposition. (a) Comparison of analytical signal and its reconstructed signal; lines in French blue are analytical signal; lines in Maroon are reconstructed signals using SW decomposition. (b) Table of parameters used in reconstruction.

of the signal's temporal structure. Just like the concept of frequency of a system, the newly introduced parameter $\kappa$ is an intrinsic characteristic of a signal, which can be used to differentiate or correlate various signals, and to trace the change of SW components of a signal. Although we only provided cases in the fields of heart sound analysis, seismic characterization and gravitational wave physics here, we have checked the effective applications of SW and decomposition method in other engineering fields, which include, but are not limited to, the signal used in hyperbolic radio navigation system (Loran-C) [9], auditory and acoustic signal processing[27] and mechanical shock and pyroshock[10]. Therefore, we expect that the proposed SW decomposition is an efficient general tool for the analyses of signals associated with the dynamic responses of physical and engineering systems disturbed by impulsive actions. It can also largely increase the efficiency of high fidelity signal representation, data management and communication.

\section{Data and materials availability}

The facilities of PASCAL, IRIS, and GRIT Data Services were used for access to waveforms, related metadata, and/or derived products used in this study.

\section{References}

[1] ECSS-E-HB-32-25A, Mechanical Shock Design and Verification Handbook, European Space Agency, European Cooperation for Space Standardization, 14 July 2015. 
[2] S. W. Smith, et al., The scientist and engineer's guide to digital signal processing, California Technical Pub. San Diego, 1997.

[3] C. Torrence, G. P. Compo, A practical guide to wavelet analysis, Bulletin of the American Meteorological society 79 (1) (1998) 61-78.

[4] A. Aertsen, P. I. Johannesma, D. Hermes, Spectro-temporal receptive fields of auditory neurons in the grassfrog, Biological Cybernetics 38 (4) (1980) 235-248.

[5] R. D. Patterson, K. Robinson, J. Holdsworth, D. McKeown, C. Zhang, M. Allerhand, Complex sounds and auditory images, in: Auditory physiology and perception, Elsevier, 1992, pp. 429-446.

[6] A. Bell, H. P. Wit, Cochlear impulse responses resolved into sets of gammatones: the case for beating of closely spaced local resonances, PeerJ 6 (2018) e6016.

[7] D. Kern, C. Hayes, Transient vibration test criteria for spacecraft hardware, The Shock and Vibration Bulletin 54 (1984) 99-109.

[8] N. M. Newmark, Fundamentals of earthquake engineering, Printice Hall (1974).

[9] D. Wang, X. Xi, Y. Pu, J. Liu, L. Zhou, Parabolic equation method for loran-c asf prediction over irregular terrain, IEEE Antennas and Wireless Propagation Letters 15 (2016) 734-737.

[10] Y. Yan, Q. M. Li, A general shock waveform and characterisation method, submitted to Mechanical Systems and Signal Processing (in review after second revision), also available at arXiv preprint arXiv:1902.07996 (2019).

[11] S. G. Mallat, Z. Zhang, Matching pursuits with time-frequency dictionaries, IEEE Transactions on signal processing 41 (12) (1993) 3397-3415.

[12] MathWorks, Matching pursuit: MATLAB Documentation, https://ww2.mathworks.cn/help/wavelet/ref/wmpalg.html\#bs9vu3gMPDICT (Accessed 03/10/2019).

[13] S. Singh, Application of Prony analysis to characterize pulsed corona reactor measurements, MSc Dissertation, University of Wyoming, Laramie, USA (2003).

[14] R. C. Ferebee, J. Clayton, D. Alldredge, T. Irvine, An Alternative Method of Specifying Shock Test Criteria, Tech. Rep. NASA/TM-2008-215253, NASA, Huntsville, USA (2008).

[15] P. Bentley, G. Nordehn, M. Coimbra, S. Mannor, The PASCAL Classifying Heart Sounds Challenge 2011 (CHSC2011) Results, http://www.peterjbentley.com/heartchallenge/index.html (Accessed 07/09/2018).

[16] A. Leatham, Auscultation of the Heart and Phonocardiography, Churchill Livingstone, 1975.

[17] Incorporated research institutions for seismology, https://www.iris.edu (Accessed 07/09/2018).

[18] E. Berti, V. Cardoso, J. A. Gonzalez, U. Sperhake, M. Hannam, S. Husa, B. Brügmann, Inspiral, merger, and ringdown of unequal mass black hole binaries: A multipolar analysis, Physical Review D 76 (6) (2007) 064034.

[19] A. Buonanno, G. B. Cook, F. Pretorius, Inspiral, merger, and ring-down of equal-mass black-hole binaries, Physical Review D 75 (12) (2007) 124018.

[20] U. Sperhake, E. Berti, V. Cardoso, Numerical simulations of black-hole binaries and gravitational wave emission, Comptes Rendus Physique 14 (4) (2013) 306-317.

[21] E. Berti, V. Cardoso, A. O. Starinets, Quasinormal modes of black holes and black branes, Classical and Quantum Gravity 26 (16) (2009) 163001.

[22] K. D. Kokkotas, B. G. Schmidt, Quasi-normal modes of stars and black holes, Living Reviews in Relativity 2 (1) (1999) 2 .

[23] R. Konoplya, A. Zhidenko, Quasinormal modes of black holes: From astrophysics to string theory, Reviews of Modern Physics 83 (3) (2011) 793.

[24] V. Cardoso, E. Franzin, P. Pani, Is the gravitational-wave ringdown a probe of the event horizon?, Physical review letters 116 (17) (2016) 171101.

[25] Homepage of grit, https://centra.tecnico.ulisboa.pt/network/grit/files/ (Accessed 07/09/2018).

[26] V. Cardoso, P. Pani, Tests for the existence of black holes through gravitational wave echoes, Nature Astronomy 1 (9) (2017) 586.

[27] R. V. Sharan, T. J. Moir, An overview of applications and advancements in automatic sound recognition, Neurocomputing $200(2016) 22-34$

\section{Appendix A. Shock Decomposition Procedure}

This signal processing tool is proposed in Ref.[10] to decompose any given shock signal into a sum of shock waveform components. To prevent potential arithmetic overflow during exponentiation calculation, Eq.(1) is rewritten in the following form,

$$
w(t)=A e^{\zeta \omega \tau(\ln t-\ln \tau)+\zeta \omega(\tau-t)+i(\omega t+\varphi)} H(t)
$$

Generally, the main idea of shock decomposition algorithm as shown in Fig.A.6, is to fit a series of shock waveform components described by Eq.(A.1) to a shock signal. The energy ratio $\epsilon$ of a waveform component $\left(w_{i}\right)$ is calculated in Eq.(A.2) to evaluate its weight of contribution,

$$
\epsilon_{w i}=\frac{E_{w i}}{E_{r}}
$$




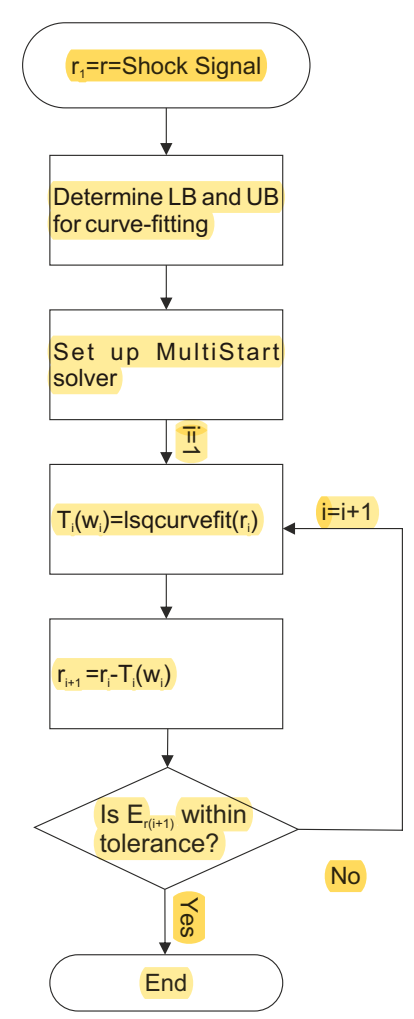

Figure A.6: Flowchart of shock decomposition method

where $E_{w i}$ is the signal energy of $w_{i}$ calculated by

$$
E_{w i}=\left\langle w_{i}(t), w_{i}(t)\right\rangle=\int_{-\infty}^{\infty}\left|w_{i}(t)\right|^{2} d t
$$

and $E_{r}$ is the signal energy of shock signal $r$.

The first shock waveform component $T_{1}\left(w_{1}\right)$ can be obtained by fitting the original shock signal $(r)$, i.e., $r_{1}=r$. The residue, $r_{2}$, comes from separating the first shock waveform component from the original shock signal by $r_{2}=r_{1}-T_{1}\left(w_{1}\right)$, whose energy ratio $\epsilon_{r 2}$ is still not negligible. This non-linear curve fitting problem

$$
\min _{\boldsymbol{x}_{1}}\left(E_{r 2}\right)=\min _{\boldsymbol{x}_{1}} \int_{-\infty}^{\infty}\left|r_{1}(t)-T_{1}\left(w_{1}(t)\right)\right|^{2} d t
$$

is solved in least-squares sense with 'Isqcurvefit' algorithm in Matlab, where $\boldsymbol{x}_{1}$ is the solution vector of all parameters to be calculated.

$$
\boldsymbol{x}_{1}=\left[A_{1}, \omega_{1}, \grave{t}_{1}, \tau_{1}, \zeta_{1}, \varphi_{1}\right]
$$

There may be many local minima of this non-linear problem, in contrast to linear curve fitting where the local minima is also the global minima. The global solution can be found by using various starting points $\left(\stackrel{\circ}{x}_{1}\right)$ for the fitting, which is achieved by 'MultiStart' strategy in Matlab. The lower bound (LB) and upper bound (UB) of fitting parameters can be set according to the mechanical condition of each shock signal, e.g., peak amplitude, interesting frequency range, etc. These starting points are discrete combination of parameters in the feasible region defined by LB and UB.

Normally $\epsilon_{w 1}$ can account for the main proportion of the shock signal $r$, but the energy ratio $\epsilon_{r 2}$ still accounts for non-negligible energy. Hence $r_{2}$ is treated as the new curve to be fitted. This non-linear fitting 
procedure can be repeated for all the subsequent residues $r_{i}(i \geqslant 2)$, with $r_{i+1}=r_{i}-T_{i}\left(w_{i}\right)$.

$$
\begin{gathered}
r 2=r 1-T_{1}\left(w_{1}\right) \\
r 3=r 2-T_{2}\left(w_{2}\right) \\
\vdots \\
r_{i+1}=r_{i}-T_{i}\left(w_{i}\right) \\
r_{i+1}=r-\sum_{j=1}^{i} T_{j}\left(w_{j}\right)
\end{gathered}
$$

thus,

According to Eqs.(A.6) and (A.7), $r_{i+1}$ is the global error between the shock signal and the reconstructed signal. The decomposition procedure finally stops when the energy ratio $\epsilon_{r(i+1)}$ of the global error $r_{i+1}$ is within the acceptable tolerance, which is $10 \%$ in this study. 